\title{
Erratum to: Strategic Innovative Marketing
}

\author{
Androniki Kavoura, Damianos P. Sakas and Petros Tomaras
}

\section{Erratum to:}

\section{A. Kavoura et al. (eds.), Strategic Innovative Marketing, Springer Proceedings in Business and Economics, DOI 10.1007/978-3-319-56288-9}

In the original version of the book, the following belated corrections to change the order of First name and Family name of chapter authors have to be incorporated:

In Chapter 19, Platis Charalampos and Papadaki Maria have to be changed to read as Charalampos Platis and Maria Papadaki.

In Chapter 21, Zoulias Emmanouil has to be changed to read as Emmanouil Zoulias.

In Chapter 23, Papadimitriou Athanasia has to be changed to read as Athanasia Papadimitriou.

In Chapter 32, Kontis Alexios-Patapios and Gkoumas Aristeidis have to be changed to read as Alexios-Patapios Kontis and Aristeidis Gkoumas.

In Chapter 56, Apostolos P. Sarlis has to be changed to read as Apostolos S. Sarlis.

The erratum book has been updated with the changes.

The updated online version of this book can be found at

http://dx.doi.org/10.1007/978-3-319-56288-9

http://dx.doi.org/10.1007/978-3-319-56288-9_19

http://dx.doi.org/10.1007/978-3-319-56288-9_21

http://dx.doi.org/10.1007/978-3-319-56288-9_23

http://dx.doi.org/10.1007/978-3-319-56288-9_32

http://dx.doi.org/10.1007/978-3-319-56288-9_56 